\title{
BingleSeq: A user-friendly $R$ package for bulk and single-cell RNA-Seq data analysis
}

\author{
Daniel Dimitrov ${ }^{1}$, Quan Gu ${ }^{\text {Corresp. } 1}$ \\ 1 MRC-University of Glasgow Centre for Virus Research, University of Glasgow, Glasgow, United Kingdom \\ Corresponding Author: Quan Gu \\ Email address: quan.gu@glasgow.ac.uk
}

\section{Background}

RNA sequencing is an indispensable research tool used in a broad range of transcriptome analysis studies. The most common application of RNA Sequencing is differential expression analysis and it is used to determine genetic loci with distinct expression across different conditions. An emerging field called single-cell RNA sequencing is used for transcriptome profiling the individual cell level. The standard protocols for both of these approaches include the processing of sequencing libraries and result in the generation of count matrices. An obstacle to these analyses and the acquisition of meaningful results is that they require programming expertise. Although some effort has been directed toward the development of user-friendly RNA-Seq analysis analysis tools, few have the flexibility to explore both Bulk and single-cell RNA sequencing.

\section{Implementation}

BingleSeq was developed as an intuitive application that provides a user-friendly solution for the analysis of count matrices produced by both Bulk and Single-cell RNA-Seq experiments. This was achieved by building an interactive dashboard-like user interface which incorporates three state-of-the-art software packages for each type of the aforementioned analyses. Furthermore, BingleSeq also includes additional features such as visualisation techniques, extensive functional annotation analysis and rank-based consensus for differential gene analysis results. As a result, BingleSeq puts some of the best reviewed and most widely used packages and tools for RNA-Seq analyses at the fingertips of biologists with no programming experience.

\section{Availability}

BingleSeq is as an easy-to-install R package available on GitHub at https://github.com/dbdimitrov/BingleSeq/ 
1

2 BingleSeq: A user-friendly R package for bulk and

3 single-cell RNA-Seq data analysis

4

5 Daniel Dimitrov ${ }^{1}$, Quan $\mathrm{Gu}^{1, *}$

$6{ }^{1}$ MRC-University of Glasgow Centre for Virus Research, University of Glasgow, Glasgow,

7 United Kingdom

8

$9{ }^{*}$ Corresponding Author: Quan $\mathrm{Gu}^{1}$

10464 Bearsden Road, Glasgow, G61 1QH, United Kingdom

11 Email address: quan.gu@glasgow.ac.uk

\section{Abstract}

\section{Background}

RNA sequencing is an indispensable research tool used in a broad range of transcriptome analysis studies. The most common application of RNA Sequencing is differential expression analysis and it is used to determine genetic loci with distinct expression across different conditions. An emerging field called single-cell RNA sequencing is used for transcriptome profiling at the individual cell level. The standard protocols for both of these approaches include the processing of sequencing libraries and result in the generation of count matrices. An obstacle to these analyses and the acquisition of meaningful results is that they require programming expertise. Although some effort has been directed toward the development of user-friendly RNA-Seq analysis analysis tools, few have the flexibility to explore both Bulk and single-cell

RNA sequencing.

\section{Implementation}

BingleSeq was developed as an intuitive application that provides a user-friendly solution for the analysis of count matrices produced by both Bulk and Single-cell RNA-Seq experiments. This was achieved by building an interactive dashboard-like user interface which incorporates three state-of-the-art software packages for each type of the aforementioned analyses. Furthermore, BingleSeq also includes additional features such as visualisation techniques, extensive functional annotation analysis and rank-based consensus for differential expression analysis results. As a result, BingleSeq puts some of the best reviewed and most widely used packages and tools for RNA-Seq analyses at the fingertips of biologists with no programming experience. 
40

41

42

43

44

45

46

47

48

49

50

51

52

53

54

55

56

57

58

59

60

61

62

63

64

65

66

67

68

69

70

71

72

73

74

75

76

77

78

79

\section{Availability}

BingleSeq is as an easy-to-install $\mathrm{R}$ package available on GitHub at https:/github.com/dbdimitrov/BingleSeq/

\section{Introduction}

About a decade ago, a transcriptome profiling approach, known as RNA Sequencing (RNA-Seq), was predicted to revolutionize transcriptome analyses (Wang et al., 2009). Today, as a consequence of the continuous advancements and dropping costs of next-generation sequencing (NGS) technologies, differential expression (DE) analysis or Bulk RNA-Seq has established itself as a routine research tool (Stark et al., 2019).

Single-cell RNA Sequencing (scRNA-Seq) is an emerging field that enables the gene expression profiling at the individual cell level and it is believed to lead to the reconstruction of an entire human cell lineage tree (Shapiro et al., 2013). scRNA-Seq's potential is highlighted by its broad range of applications which include the classification and profiling of subpopulations of cells to putative transcriptomic types (Jaitin et al., 2014; Macosko et al., 2015; Muraro et al., 2016), the discovery of novel cell types (Campbell et al., 2017; Villani et al., 2017) and novel microbial species (Rinke et al., 2013), as well as the deconvolution of Bulk RNA-Seq results (Schelker et al., 2017; Tirosh et al., 2016).

Although there is a wide range of software tools available for both Bulk RNA-Seq and scRNASeq analyses, most require some proficiency in programming languages such as $\mathrm{R}$. This creates a challenge for the analysis of RNA-seq data for a large portion of biologists lacking programming experience. Here we present an application, called BingleSeq, the primary goal of which is to enable the user-friendly analysis of count tables obtained by both Bulk RNA-Seq and scRNASeq protocols.

\section{Materials \& Methods}

Implementation. BingleSeq is based on shiny (Chang et al., 2016) and it is composed of a multi-tabbed UI, built as separate shiny modules with efficiency, code readability and reusability in mind. Each module (tab) corresponds to a key step in the typical Bulk and scRNA-Seq analysis pipelines (Fig. 1). Modules are generated only upon reaching a given step of the analyses which ensures efficiency and speed despite the complexity of the application. BingleSeq's UI components (e.g. plots, tables, tabs, etc.) make use of shiny's 'reactivity' property and these components are automatically updated upon user input or any change related to a given component.

The development of BingleSeq focused on providing a flexible and intuitive user experience. As 
80

81

82

83

84

85

86

87

88

89

90

91

92

93

94

95

96

97

98

99

100

101

102

103

104

105

106

107

108

109

110

111

112

113

114

115

116

117

118

119

such, BingleSeq implements three state-of-the art package options for each analysis, while it also enables users to tailor key analysis parameters according to their experiment.

User experience is complemented with customizable and interactive tables and plots as well as analysis-related tips and pop-up messages to guide the correct execution of the pipelines. Plot interactivity was achieved via conversion from ggplot to ggplotly (Sievert, 2020).

Bulk RNA-Seq Steps and Features. To begin the DE analysis of Bulk RNA-Seq data, a count table and a metadata table must be loaded in the appropriate formats (Fig S1). Genes can then be filtered according to counts per million (CPM), Max, or Median thresholds. Furthermore, batch effect correction can be performed with Harman and ComBat packages (Leek et al., 2012; Oytam et al., 2016) (Fig S2).

Subsequent to quality control, users can investigate differentially expressed genes (DEGs) using three state-of-the-art packages: DESeq2 (Love et al., 2014), edgeR (Robinson et al., 2010), and limma (Ritchie et al., 2015). Upon obtaining DE results, users can visualize them using interactive plotting techniques (Fig. 2A-E). These include: a PCA plot used to provide insights about the relationship between samples; Barchart plot supplemented with a summary table used to summarize the up- and downregulated DEGs; Volcano and MA plots that assess the relationship between fold change (FC) versus significance and average expression; and Heatmaps which are arguably the most versatile and informative type of visualization technique when looking at $\mathrm{DE}$ results. BingleSeq's visualization techniques were implemented with customization in mind and users can specify parameters such as p-value threshold and fold-change threshold, among others. Due to their versatility, heatmaps were designed as BingleSeq's most customizable plotting component (Fig. 2F).

To assess BingleSeq's Bulk RNA-Seq pipeline, we used a synthetic dataset generated with the compcodeR package (Soneson, 2014). We also tested it on a real data set looking at DEGs between HSV-1 infected control and interferon B treatment (McFarlane et al., 2019) - see Article S1.

scRNA-Seq Pipeline Steps and Features. BingleSeq's scRNA-Seq part is based on Seurat's scRNA-Seq pipeline and visualizations (Satija et al., 2015). Nonetheless, clustering can also be performed with monocle (Trapnell et al., 2014) and SC3 (Kiselev et al., 2017) packages. To begin scRNA-Seq analysis, data can be supplied in two formats - 'Cell Ranger 10x Genomics' data and a count table in a predefined format (Fig S3). Once the data is loaded, users can filter unwanted cells and features (Fig S4). The next step is to normalize the data and BingleSeq provides two Seurat-supplied normalization methods - "LogNormalize" and "Relative counts". Simultaneously with normalization and scaling, the highly variable features within the dataset are identified and selected for clustering with Seurat as a way to minimize noise. The highly variable features are visualized using an interactive scatter plot (Fig S4). 
120 Following normalization, the 'Clustering' tab is generated (Fig. 3) which provides a high degree

121 of control over the different steps of the analysis (Fig. 3A). Additionally, general tips are

122 provided, such as clustering advice provided for each package (Fig. 3B). Moreover, each of the

123 visualisation techniques in this tab are interactive to further facilitate the interpretation of results.

124 First, the clustering tab requires the generation of pre-clustering prerequisites such as scaling the

125 data and dimensionality reduction with Principal Component Analysis (PCA).

126 Then an elbow plot is returned which is used to determine the dimensionality of the dataset (Fig.

127 3C) - this is essential for excluding noise when clustering with Seurat and monocle. PC

128 heatmaps (Fig. 3D) are also available as a further tool for PC Selection.

129 Once the data is filtered and transformed, users can proceed to unsupervised clustering with

130 Seurat, $\mathrm{SC} 3$, and monocle. The primary way to visualize clustering results is via t-distributed

131 stochastic neighbour embedding (tSNE) plots (Fig. 3E-G) - a method designed for the purpose

132 of visualizing high dimensional datasets (Maaten and Hinton, 2008).

133 Following clustering, DE analysis can be conducted using Seurat's inbuilt testing methods to

134 identify marker genes. The implemented Seurat DE methods include: Student's T test, Wilcoxon

135 Rank Sum test, DESEq2 (Love et al., 2014), and MAST package (Finak et al., 2015). DE results

136 and specific marker genes can then be visualized using Seurat's inbuilt plots. These plots include

137 a cluster heatmap and visualizations for the exploration of specific genes via Violin, Feature, and

138 Ridge plots (Fig. 4).

139 An evaluation of BingleSeq's scRNA-Seq pipeline was performed by reproducing and extending

140 the results of Seurat's online tutorial (https://satijalab.org/seurat/v3.0/pbmc3k tutorial.html) - see

141 Article S2. The tutorial is based on a 10x Genomics dataset of 2700 Peripheral Blood

142 Mononuclear Cells (PBMCs) with $\sim 69,000$ reads per cell. The data set is available at

143 https://support.10xgenomics.com/single-cell-gene-expression/datasets/1.1.0/pbmc3k.

144

145 Functional Annotation. For both Bulk and scRNA-Seq pipelines, BingleSeq enables the

146 functional annotation of results using GOseq (Young et al., 2010) and transcription factor (TF)

147 and pathway activity inference tools DoRothEA and PROGENy (Schubert et al., 2018; Garcia-

148 Alonso, et al., 2019; Holland et al., 2019, Holland et al., 2020).

149 GOSeq is implemented in the 'Gene Ontology' tab and it enables users to obtain results from

150 KEGG pathway analysis and three types of GO categories, including 'Cellular Component',

151 'Molecular Function', and 'Biological Function' (Fig S5). The 'Gene Ontology' tab can also be

152 used to generate top $10 \mathrm{GO}$ term histograms and to obtain additional information about a given

153 GO term using the 'GO.db' package (Carlson et al., 2019) (Fig S5). Note that BingleSeq

154 supports human, mouse, drosophila, zebrafish, and Escherichia coli $\mathrm{K} 12$ strain genomes

155 (Carlson, 2019a-d) for analysis with GOSeq.

156 In BingleSeq, the gene set resource DoRothEA is coupled to the statistical method viper

157 (Alvarez et al., 2016) and together they enable the inference of TF activity (Fig S6A-B). On the

158 other hand, PROGENy is used to estimate the activity of 14 signalling pathways from gene

159 expression using a linear model (Fig S6C-D). DoRothEA and PROGENy are footprint-based 
160 tools and they infer activity from the expression of molecules considered to be downstream of a

161

162

163

164

165

166

167

168

169

170

171

172

173

174

175

176

177

178

179

180

181

182

183

184

185

186

187

188

189

190

191

192

193

194

195

196

197

198

199

given TF or pathway, respectively. Both of these tools are implemented in the 'Footprint

Analysis' tab and are available for human and mouse data.

DE Package Comparison and Rank-Based Consensus. BingleSeq supplies an option to assess the agreement between the different DE analysis methods in the form of a Venn diagram (Fig S7A). Moreover, BingleSeq provides a rank-based consensus approach to improve confidence in DE results (Costa-Silva et al., 2017; Guo et al., 2014; Moulos and Hatzis, 2015) - see Fig S7B. In the context of the Bulk RNA-Seq pipeline the overlap in DEGs is assessed on the results obtained using DESeq2, edgeR, and limma packages. In the case of scRNA-Seq, this is done using three of Seurat's inbuilt DE methods - MAST, Wilcoxon Rank Sum Test, and Student's T test.

Inbuilt bulk and single cell RNA-Seq example datasets. BingleSeq also provides inbuilt Bulk RNA-Seq and scRNA-Seq test data. Bulk RNA-Seq data is represented by a 3-sample contrast between HSV-1 infected control and interferon B treatment (McFarlane et al., 2019). The data files are available from European Nucleotide Archive (ENA) under accession number PRJEB27501. The single-cell RNA-Seq example is a Cell Ranger 10x Genomics dataset looking at filtered data of 2700 human peripheral blood mononuclear cells.

\section{Results}

Some effort has already been directed towards lowering the entry requirements to RNA-Seq analyses as there are some software tools which implement UI components. However, many of these applications are limited to only some key features or particular parts of RNA-Seq analysis (DeTomaso and Yosef, 2016; Kiselev et al., 2017).

From the available software packages providing comprehensive solutions for Bulk RNA-Seq, NetworkAnalyst (Zhou et al., 2019), DEapp (Li and Andrade, 2017), DEBrowser (Kucukural et al., 2019), and Omics Playground (Akhmedov et al., 2020) were thought to provide the most extensive RNA-Seq analysis options. As seen in Table S1-2, the functionality implemented BingleSeq's Bulk RNA-Seq can be argued to put our package close to even the best available Bulk RNA-Seq solutions.

When looking at similar applications providing solutions to scRNA-Seq analysis, BingleSeq is most analogous to SeuratWizard (Yousif et al., 2019) as both are based on Seurat's pipeline. However, by implementing SC3 and monocle, BingleSeq provides solutions to some of Seurat's inherent limitations. For instance, SeuratWizard does not implement functionality to explicitly specify the number of clusters, nor a way to estimate the number of clusters, while BingleSeq provides two distinct approaches to achieve that. Another major functionality that sets our 
200

201

202

203

204

205

206

207

208

209

210

211

212

213

214

215

216

217

218

219

220

221

222

223

224

225

226

227

228

229

230

231

232

233

234

235

236

237

238

application apart is that it enables functional annotation analysis and a way to compare and provide a consensus for Seurat's inbuilt DE methods. Consequently, BingleSeq can be argued to match even the most comprehensive scRNA-Seq applications, such as ASAP (Gardeux et al., 2017) and singleCellTK (Jenkins et al., 2019) - see Table S2-3.

In terms of providing a solution to both Bulk and single-cell RNA-Seq analyses, BingleSeq's features and comprehensiveness are only contested by those of Omics Playground. However, what sets BingleSeq apart is that it provides multiple clustering packages and algorithms.

\section{Discussion}

As solutions to Bulk RNA-Seq, BingleSeq implements DESeq2, edgeR, and limma. These packages are well-tested and regarded as being among the best performing ones (Schurch et al., 2016; Seyednasrollah et al., 2015; Soneson and Delorenzi, 2013). Despite being accepted as being among the best DE analysis solutions, different studies often present contrasting conclusions. Hence, there is little consensus regarding which DE algorithm has the best performance. This stems from the fact that there is no optimal package under all circumstances and different variables are known to affect package performance, with sample size in particular (Schurch et al., 2016; Soneson and Delorenzi, 2013). As such, the method of choice depends on the dataset being analysed.

BingleSeq's scRNA-Seq pipeline includes three unsupervised clustering solutions provided by monocle, Seurat, and SC3 packages. The latter two packages are regarded as having the best overall clustering performance (Duò et al., 2018; Freytag et al., 2018). However, similarly to packages used in the DE analysis of Bulk RNA-Seq data, there seems to be little consensus on which package provides the best-performing clustering approach. This is largely due to the inherent limitations of the different algorithms used in clustering, as a result no algorithm performs well in every circumstance (Wiwie et al., 2015). Kiselev et al. (2019) suggest that Seurat may be inappropriate for small scRNA-Seq datasets, due to the inherent limitations of the Louvain algorithm. On the contrary, as a way to amend for the limitations of k-means clustering algorithm used in SC3, the authors implemented an extensive iterative-consensus approach, which makes SC3 magnitudes slower than Seurat and downgrades its scalability (Duò et al., 2018; Kiselev et al., 2019). Another difference between these two packages is that Seurat does not include functionality to estimate or explicitly specify cluster number, while SC3 does.

Thus, by implementing multiple DE and clustering packages BingleSeq enables users to explore and pick the method that is most suitable for their experiment. 
239

240

241

242

243

244

245

246

247

248

249

250

251

252

253

254

255

256

257

258

259

260

261

262

263

264

265

266

267

268

269

270

271

272

273

274

275

276

277

278

BingleSeq is a comprehensive and intuitive solution that enables users to choose from multiple state-of-the-art DE analysis and unsupervised clustering packages according to their preferences or the dataset in question. In terms of Bulk RNA-Seq analyses, BingleSeq implements functionality that puts it close to, what are to our understanding, the best available similar applications. In terms of scRNA-Seq, BingleSeq could be argued to be among the most exhaustive applications, such as ASAP (Gardeux et al., 2017) and singleCellTK (Jenkins et al., 2019). Thus, the implementation of multiple state-of-the-art packages for both types of analysis, alongside functionality that bolsters the confidence and subsequent interpretation of DE results, makes BingleSeq a solid choice for the analysis of both Bulk and scRNA-Seq.

Future work will focus on including more functional annotation options and on extending user control over the packages implemented in BingleSeq. Moreover, the implementation of both Bulk RNA-Seq and scRNA-Seq pipelines puts BingleSeq in a particularly good position to implement user-friendly deconvolution of Bulk RNA-Seq results using scRNA-Seq data. Hence, an excellent and practical conclusion to the development of BingleSeq would be to include stateof-the-art deconvolution methods such as Cell Population Mapping (CPM) (Frishberg et al., 2019) or MUlti-Subject SIngle Cell deconvolution (MuSiC) (Wang et al., 2019).

BingleSeq is as an easy-to-install R package available on GitHub at https://github.com/dbdimitrov/BingleSeq/. The application's GitHub page provides an easy guide on how to install the application as well as examples of its general applicability and an extensive description of typical workflows when working with Bulk RNA-Seq and scRNA-Seq data.

\section{Acknowledgments}

We acknowledge the financial support for bioinformatics developments as part of MRC (MC_UU_12014/12). We also extend our gratitude to Sejal Modha, Joseph Hughes, Richard Orton, Srikeerthana Kuchi, and Martín Garrido-Rodríguez for reviewing the manuscript prior to submission.

\section{References}

Akhmedov, M., Martinelli, A., Geiger, R., Kwee, I., 2020. Omics Playground: a comprehensive self-service platform for visualization, analytics and exploration of Big Omics Data.

NAR Genom Bioinform 2. https://doi.org/10.1093/nargab/lqz019

Alvarez MJ, Shen Y, Giorgi FM, Lachmann A, Ding BB, Ye BH, Califano A (2016).

"Functional characterization of somatic mutations in cancer using network-based inference of protein activity." Nature genetics, 48(8), 838-47.

PeerJ reviewing PDF | (2020:07:51225:1:1:NEW 17 Oct 2020) 
279

280

281

282

283

284

285

286

287

288

289

290

291

292

293

294

295

296

297

298

299

300

301

302

303

304

305

306

307

308

309

310

311

312

313

314

315

316

Campbell, J.N., Macosko, E.Z., Fenselau, H., Pers, T.H., Lyubetskaya, A., Tenen, D., Goldman, M., Verstegen, A.M.J., Resch, J.M., McCarroll, S.A., Rosen, E.D., Lowell, B.B., Tsai, L.T., 2017. A molecular census of arcuate hypothalamus and median eminence cell types. Nat Neurosci 20, 484-496. https://doi.org/10.1038/nn.4495

Carlson, M., 2019. org. Hs. eg. db: Genome Wide Annotation for E coli strain K12. R package version 3.8.2. https://doi.org/10.18129/B9.bioc.org.EcK12.eg.db

Carlson, M., 2019. org. Hs. eg. db: Genome Wide Annotation for Fly. R package version 3.8.2. https://doi.org/10.18129/B9.bioc.org.Dm.eg.db

Carlson, M., 2019. org. Hs. eg. db: Genome Wide Annotation for Human. R package version 3.8.2. https://doi.org/10.18129/B9.bioc.org.Hs.eg.db

Carlson, M., n.d. org. Mm. eg. db: Genome wide annotation for Mouse. R package version 3.8.2. Bioconductor. https://doi.org/10.18129/B9.bioc.org.Mm.eg.db

Carlson, M., 2019. org. Hs. eg. db: Genome Wide Annotation for Zebrafish. R package version 3.8.2. https://doi.org/10.18129/B9.org.Dr.eg.db

Carlson, M., Falcon, S., Pages, H., Li, N., 2019. A set of annotation maps describing the entire Gene Ontology. R package version 3. https://doi.org/10.18129/B9.bioc.GO.db

Chang, W., Cheng, J., Allaire, J.J., Xie, Y., McPherson, J., 2016. shiny: Web Application Framework for R. R package version 0.13. 2. URL: http://CRAN. R-project. org $/$ package $=$ shiny.

Costa-Silva, J., Domingues, D., Lopes, F.M., 2017. RNA-Seq differential expression analysis: An extended review and a software tool. PloS one 12.

DeTomaso, D., Yosef, N., 2016. FastProject: a tool for low-dimensional analysis of single-cell RNA-Seq data. BMC Bioinformatics 17, 315. https://doi.org/10.1186/s12859-016-1176-5

Duò, A., Robinson, M.D., Soneson, C., 2018. A systematic performance evaluation of clustering methods for single-cell RNA-seq data. F1000Res 7. https://doi.org/10.12688/f1000research.15666.2

Finak, G., McDavid, A., Yajima, M., Deng, J., Gersuk, V., Shalek, A.K., Slichter, C.K., Miller, H.W., McElrath, M.J., Prlic, M., Linsley, P.S., Gottardo, R., 2015. MAST: a flexible statistical framework for assessing transcriptional changes and characterizing heterogeneity in single-cell RNA sequencing data. Genome Biology 16, 278. https://doi.org/10.1186/s13059-015-0844-5

Freytag, S., Tian, L., Lönnstedt, I., Ng, M., Bahlo, M., 2018. Comparison of clustering tools in R for medium-sized 10x Genomics single-cell RNA-sequencing data. F1000Res 7. https://doi.org/10.12688/f1000research.15809.2

Frishberg, A., Peshes-Yaloz, N., Cohn, O., Rosentul, D., Steuerman, Y., Valadarsky, L., Yankovitz, G., Mandelboim, M., Iraqi, F.A., Amit, I., Mayo, L., Bacharach, E., Gat-Viks, I., 2019. Cell composition analysis of bulk genomics using single-cell data. Nat Methods 16, 327-332. https://doi.org/10.1038/s41592-019-0355-5 
317 Garcia-Alonso L, Holland CH, Ibrahim MM, Turei D, Saez-Rodriguez J. "Benchmark and

318

319

320

321

322

323

324

325

326

327

328

329

330

331

332

333

334

335

336

337

338

339

340

341

342

343

344

345

346

347

348

349

350

351

352

353

354

355

356 integration of resources for the estimation of human transcription factor activities." Genome Research. 2019. DOI: 10.1101/gr.240663.118.

Gardeux, V., David, F.P.A., Shajkofci, A., Schwalie, P.C., Deplancke, B., 2017. ASAP: a webbased platform for the analysis and interactive visualization of single-cell RNA-seq data. Bioinformatics 33, 3123-3125. https://doi.org/10.1093/bioinformatics/btx337

Guo, Y., Zhao, S., Ye, F., Sheng, Q., Shyr, Y., 2014. MultiRankSeq: Multiperspective Approach for RNAseq Differential Expression Analysis and Quality Control [WWW Document]. BioMed Research International. https://doi.org/10.1155/2014/248090

Jaitin, D.A., Kenigsberg, E., Keren-Shaul, H., Elefant, N., Paul, F., Zaretsky, I., Mildner, A., Cohen, N., Jung, S., Tanay, A., Amit, I., 2014. Massively Parallel Single-Cell RNA-Seq for Marker-Free Decomposition of Tissues into Cell Types. Science 343, 776-779. https://doi.org/10.1126/science.1247651

Jenkins, D.F., Faits, T., Khan, M.M., Briars, E., Pro, S.C., Johnson, W.E., 2019. singleCellTK: Interactive analysis of single cell RNA-seq data. R package version 1.

Holland C, Szalai B, Saez-Rodriguez J (2019). "Transfer of regulatory knowledge from human to mouse for functional genomics analysis." BBAGRM. Doi: 10.1016/j.bbagrm.2019.194431.

Holland C, Tanevski J, Perales-Patón J, Gleixner J, Kumar M, Mereu E, Joughin B, Stegle O, Lauffenburger D, Heyn H, Szalai B, Saez-Rodriguez J (2020). "Robustness and applicability of transcription factor and pathway analysis tools on single-cell RNA-seq data." Genome Biology. doi: 10.1186/s13059-020-1949-z.

Kiselev, V.Y., Andrews, T.S., Hemberg, M., 2019. Challenges in unsupervised clustering of single-cell RNA-seq data. Nat Rev Genet 20, 273-282. https://doi.org/10.1038/s41576018-0088-9

Kiselev, V.Y., Kirschner, K., Schaub, M.T., Andrews, T., Yiu, A., Chandra, T., Natarajan, K.N., Reik, W., Barahona, M., Green, A.R., Hemberg, M., 2017. SC3: consensus clustering of single-cell RNA-seq data. Nat Methods 14, 483-486. https://doi.org/10.1038/nmeth.4236

Kucukural, A., Yukselen, O., Ozata, D.M., Moore, M.J., Garber, M., 2019. DEBrowser: interactive differential expression analysis and visualization tool for count data. BMC Genomics 20, 6. https://doi.org/10.1186/s12864-018-5362-x

Leek, J.T., Johnson, W.E., Parker, H.S., Jaffe, A.E., Storey, J.D., 2012. The sva package for removing batch effects and other unwanted variation in high-throughput experiments. Bioinformatics 28, 882-883. https://doi.org/10.1093/bioinformatics/bts034

Li, Y., Andrade, J., 2017. DEApp: an interactive web interface for differential expression analysis of next generation sequence data. Source Code for Biology and Medicine 12, 2. https://doi.org/10.1186/s13029-017-0063-4

Love, M.I., Huber, W., Anders, S., 2014. Moderated estimation of fold change and dispersion for RNA-seq data with DESeq2. Genome Biol 15, 550. https://doi.org/10.1186/s13059-014$0550-8$ 
357 358

359

360

361

362

363

364

365

366

367

368

369

370

371

372

373

374

375

376

377

378

379

380

381

382

383

384

385

386

387

388

389

390

391

392

393

394

Maaten, L. van der, Hinton, G., 2008. Visualizing Data using t-SNE. Journal of Machine Learning Research 9, 2579-2605.

Macosko, E.Z., Basu, A., Satija, R., Nemesh, J., Shekhar, K., Goldman, M., Tirosh, I., Bialas, A.R., Kamitaki, N., Martersteck, E.M., Trombetta, J.J., Weitz, D.A., Sanes, J.R., Shalek, A.K., Regev, A., McCarroll, S.A., 2015. Highly Parallel Genome-wide Expression Profiling of Individual Cells Using Nanoliter Droplets. Cell 161, 1202-1214. https://doi.org/10.1016/j.cell.2015.05.002

McFarlane, S., Orr, A., Roberts, A.P.E., Conn, K.L., Iliev, V., Loney, C., Filipe, A. da S., Smollett, K., Gu, Q., Robertson, N., Adams, P.D., Rai, T.S., Boutell, C., 2019. The histone chaperone HIRA promotes the induction of host innate immune defences in response to HSV-1 infection. PLOS Pathogens 15, e1007667. https://doi.org/10.1371/journal.ppat.1007667

Moulos, P., Hatzis, P., 2015. Systematic integration of RNA-Seq statistical algorithms for accurate detection of differential gene expression patterns. Nucleic Acids Res 43, e25e25. https://doi.org/10.1093/nar/gku1273

Muraro, M.J., Dharmadhikari, G., Grün, D., Groen, N., Dielen, T., Jansen, E., van Gurp, L., Engelse, M.A., Carlotti, F., de Koning, E.J.P., van Oudenaarden, A., 2016. A Single-Cell Transcriptome Atlas of the Human Pancreas. Cell Systems 3, 385-394.e3. https://doi.org/10.1016/j.cels.2016.09.002

Oytam, Y., Sobhanmanesh, F., Duesing, K., Bowden, J.C., Osmond-McLeod, M., Ross, J., 2016. Risk-conscious correction of batch effects: maximising information extraction from highthroughput genomic datasets. BMC Bioinformatics 17, 332. https://doi.org/10.1186/s12859-016-1212-5

Rinke, C., Schwientek, P., Sczyrba, A., Ivanova, N.N., Anderson, I.J., Cheng, J.-F., Darling, A., Malfatti, S., Swan, B.K., Gies, E.A., Dodsworth, J.A., Hedlund, B.P., Tsiamis, G., Sievert, S.M., Liu, W.-T., Eisen, J.A., Hallam, S.J., Kyrpides, N.C., Stepanauskas, R., Rubin, E.M., Hugenholtz, P., Woyke, T., 2013. Insights into the phylogeny and coding potential of microbial dark matter. Nature 499, 431-437. https://doi.org/10.1038/nature12352

Ritchie, M.E., Phipson, B., Wu, D., Hu, Y., Law, C.W., Shi, W., Smyth, G.K., 2015. limma powers differential expression analyses for RNA-sequencing and microarray studies. Nucleic Acids Res 43, e47-e47. https://doi.org/10.1093/nar/gkv007

Robinson, M.D., McCarthy, D.J., Smyth, G.K., 2010. edgeR: a Bioconductor package for differential expression analysis of digital gene expression data. Bioinformatics 26, 139140. https://doi.org/10.1093/bioinformatics/btp616

Satija, R., Farrell, J.A., Gennert, D., Schier, A.F., Regev, A., 2015. Spatial reconstruction of single-cell gene expression data. Nat Biotechnol 33, 495-502. https://doi.org/10.1038/nbt.3192 
395

396

397

398

399

400

401

402

403

404

405

406

407

408

409

410

411

412

413

414

415

416

417

418

419

420

421

422

423

424

425

426

427

428

429

430

431

432

433

434

Schelker, M., Feau, S., Du, J., Ranu, N., Klipp, E., MacBeath, G., Schoeberl, B., Raue, A., 2017. Estimation of immune cell content in tumour tissue using single-cell RNA-seq data. Nat Commun 8, 1-12. https://doi.org/10.1038/s41467-017-02289-3

Schubert M, Klinger B, Klünemann M, Sieber A, Uhlitz F, Sauer S, Garnett MJ, Blüthgen N, Saez-Rodriguez J (2018). "Perturbation-response genes reveal signaling footprints in cancer gene expression." Nature communications, 9(20).

Schurch, N.J., Schofield, P., Gierliński, M., Cole, C., Sherstnev, A., Singh, V., Wrobel, N., Gharbi, K., Simpson, G.G., Owen-Hughes, T., Blaxter, M., Barton, G.J., 2016. How many biological replicates are needed in an RNA-seq experiment and which differential expression tool should you use? RNA 22, 839-851. https://doi.org/10.1261/rna.053959.115

Seyednasrollah, F., Laiho, A., Elo, L.L., 2015. Comparison of software packages for detecting differential expression in RNA-seq studies. Brief Bioinform 16, 59-70. https://doi.org/10.1093/bib/bbt086

Shapiro, E., Biezuner, T., Linnarsson, S., 2013. Single-cell sequencing-based technologies will revolutionize whole-organism science. Nat Rev Genet 14, 618-630. https://doi.org/10.1038/nrg3542

Sievert, C. (2020). Interactive Web-Based Data Visualization with R, plotly, and shiny. Chapman and Hall/CRC. ISBN 9781138331457, https://plotly-r.com.

Soneson, C., 2014. compcodeR - an R package for benchmarking differential expression methods for RNA-seq data. Bioinformatics 30, 2517-2518. https://doi.org/10.1093/bioinformatics/btu324

Soneson, C., Delorenzi, M., 2013. A comparison of methods for differential expression analysis of RNA-seq data. BMC Bioinformatics 14, 91. https://doi.org/10.1186/1471-2105-14-91

Stark, R., Grzelak, M., Hadfield, J., 2019. RNA sequencing: the teenage years. Nat Rev Genet 20, 631-656. https://doi.org/10.1038/s41576-019-0150-2

Tirosh, I., Izar, B., Prakadan, S.M., Wadsworth, M.H., Treacy, D., Trombetta, J.J., Rotem, A., Rodman, C., Lian, C., Murphy, G., Fallahi-Sichani, M., Dutton-Regester, K., Lin, J.-R., Cohen, O., Shah, P., Lu, D., Genshaft, A.S., Hughes, T.K., Ziegler, C.G.K., Kazer, S.W., Gaillard, A., Kolb, K.E., Villani, A.-C., Johannessen, C.M., Andreev, A.Y., Allen, E.M.V., Bertagnolli, M., Sorger, P.K., Sullivan, R.J., Flaherty, K.T., Frederick, D.T., Jané-Valbuena, J., Yoon, C.H., Rozenblatt-Rosen, O., Shalek, A.K., Regev, A., Garraway, L.A., 2016. Dissecting the multicellular ecosystem of metastatic melanoma by single-cell RNA-seq. Science 352, 189-196. https://doi.org/10.1126/science.aad0501

Trapnell, C., Cacchiarelli, D., Grimsby, J., Pokharel, P., Li, S., Morse, M., Lennon, N.J., Livak, K.J., Mikkelsen, T.S., Rinn, J.L., 2014. The dynamics and regulators of cell fate decisions are revealed by pseudotemporal ordering of single cells. Nat Biotechnol 32, 381-386. https://doi.org/10.1038/nbt.2859

Villani, A.-C., Satija, R., Reynolds, G., Sarkizova, S., Shekhar, K., Fletcher, J., Griesbeck, M., Butler, A., Zheng, S., Lazo, S., Jardine, L., Dixon, D., Stephenson, E., Nilsson, E., 
435

436

437

438

439

440

441

442

443

444

445

446

447

448

449

450

451

452

453

454

455
Grundberg, I., McDonald, D., Filby, A., Li, W., Jager, P.L.D., Rozenblatt-Rosen, O., Lane, A.A., Haniffa, M., Regev, A., Hacohen, N., 2017. Single-cell RNA-seq reveals new types of human blood dendritic cells, monocytes, and progenitors. Science 356. https://doi.org/10.1126/science.aah4573

Wang, X., Park, J., Susztak, K., Zhang, N.R., Li, M., 2019. Bulk tissue cell type deconvolution with multi-subject single-cell expression reference. Nat Commun 10, 1-9. https://doi.org/10.1038/s41467-018-08023-x

Wang, Z., Gerstein, M., Snyder, M., 2009. RNA-Seq: a revolutionary tool for transcriptomics. Nat Rev Genet 10, 57-63. https://doi.org/10.1038/nrg2484

Wiwie, C., Baumbach, J., Röttger, R., 2015. Comparing the performance of biomedical clustering methods. Nat Methods 12, 1033-1038. https://doi.org/10.1038/nmeth.3583

Young, M.D., Wakefield, M.J., Smyth, G.K., Oshlack, A., 2010. Gene ontology analysis for RNA-seq: accounting for selection bias. Genome Biology 11, R14. https://doi.org/10.1186/gb-2010-11-2-r14

Yousif, A., Drou, N., Rowe, J., Khalfan, M., Gunsalus, K.C., 2019. NASQAR: A web-based platform for high-throughput sequencing data analysis and visualization. bioRxiv 709980. https://doi.org/10.1101/709980

Zhou, G., Soufan, O., Ewald, J., Hancock, R.E.W., Basu, N., Xia, J., 2019. NetworkAnalyst 3.0: a visual analytics platform for comprehensive gene expression profiling and metaanalysis. Nucleic Acids Res 47, W234-W241. https://doi.org/10.1093/nar/gkz240 
Figure 1

Overview of BingleSeq's Bulk RNA-Seq (A) and scRNA-Seq (B) analysis pipelines. 
A)

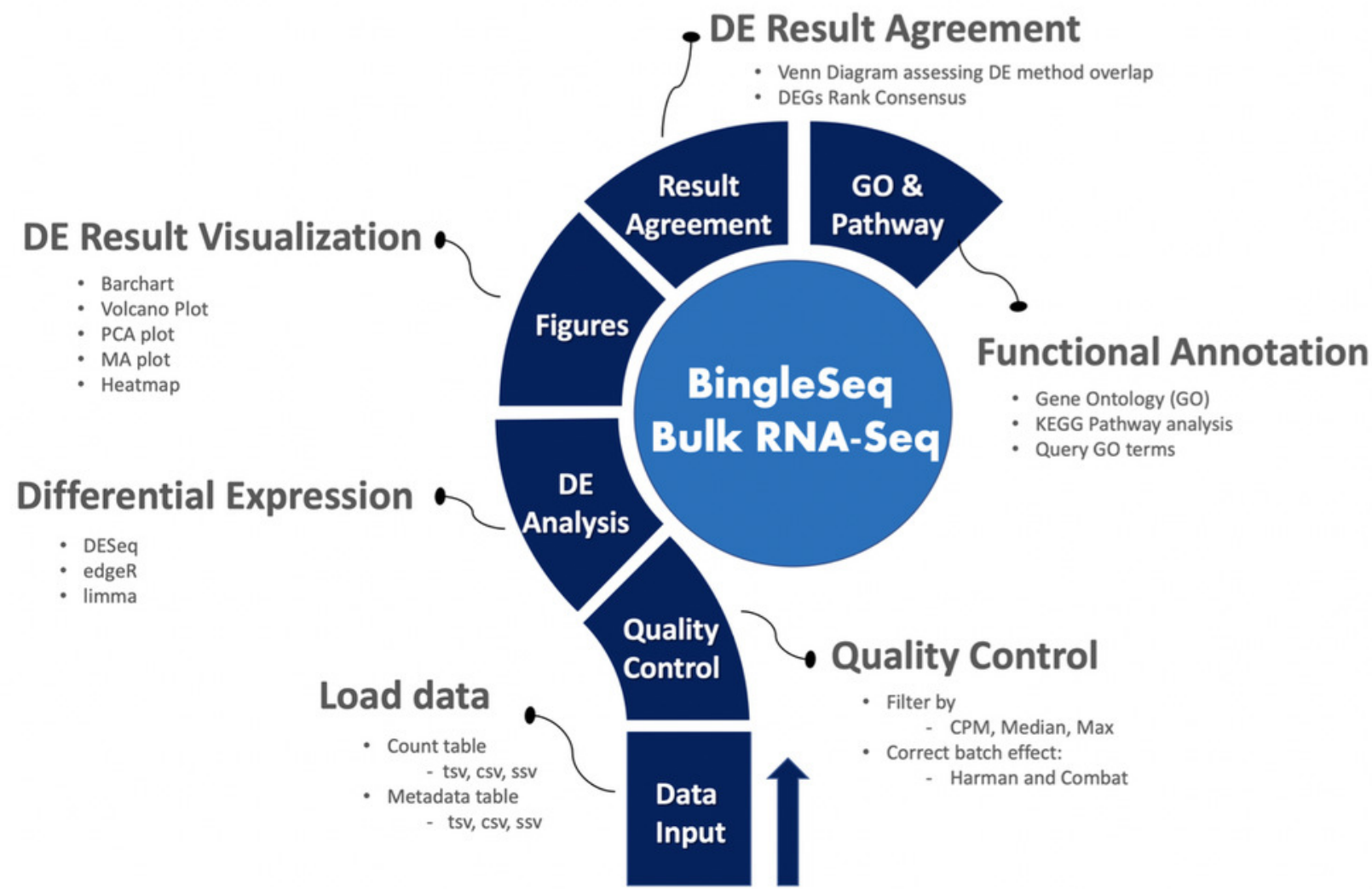

B)

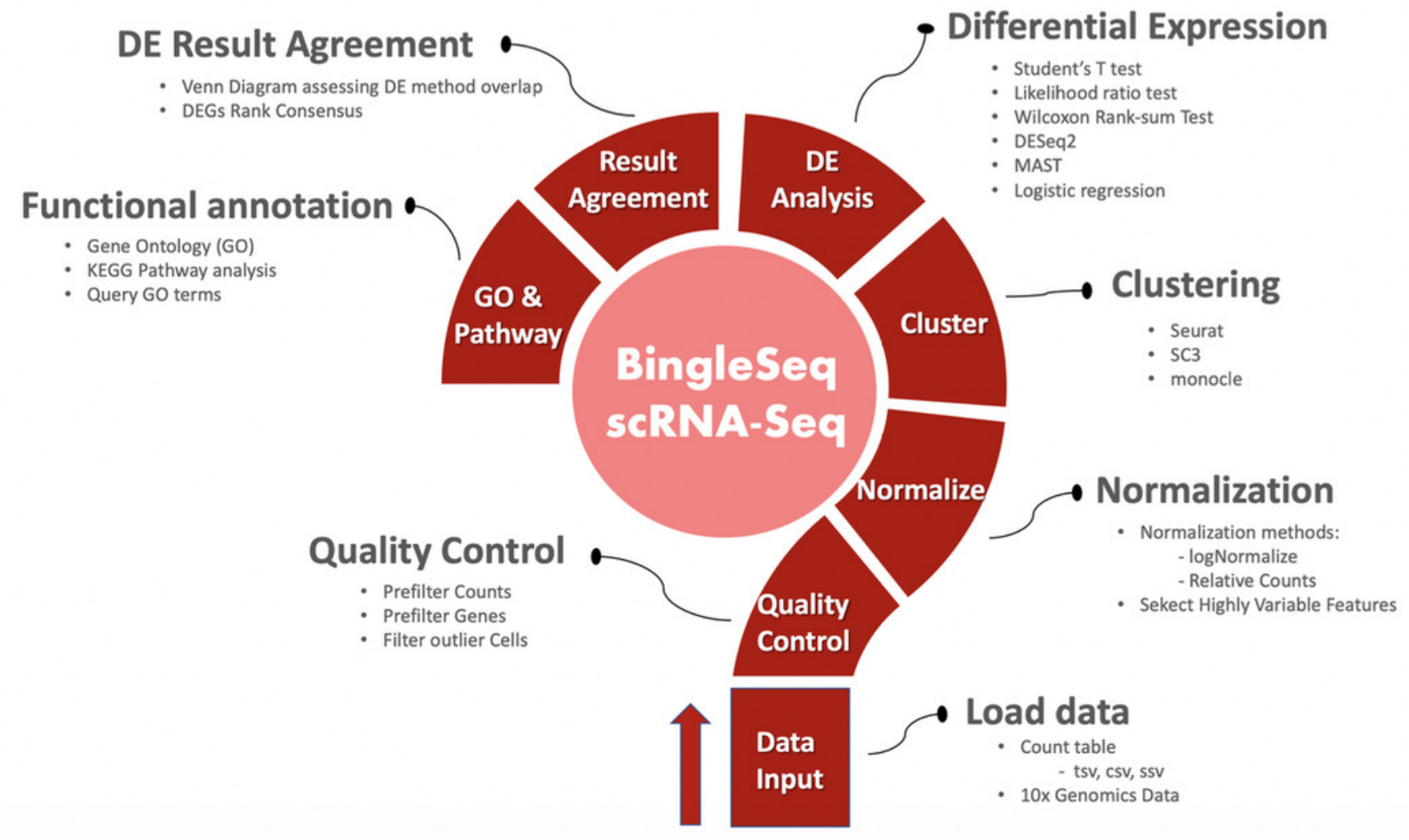




\section{Figure 2}

PCA plot (A), Barchart (B), Volcano plot (C), MA plot (D), and a Heatmap (E) with its corresponding interactive control panel $(F)$ as generated by BingleSeq.

A)

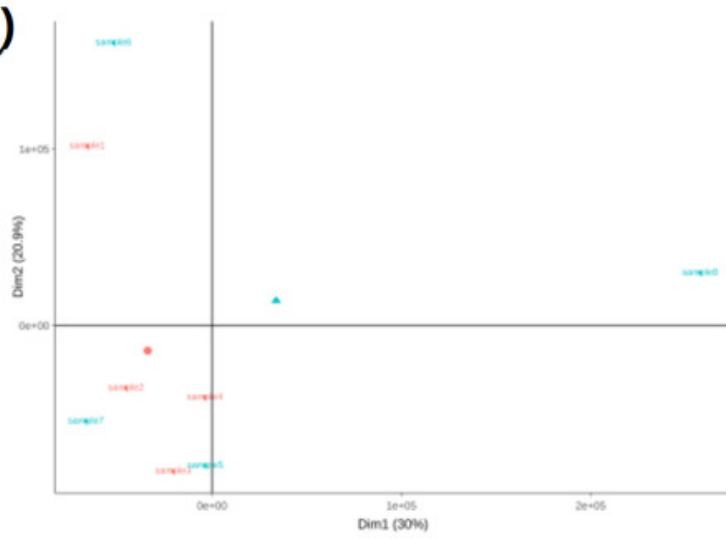

C)

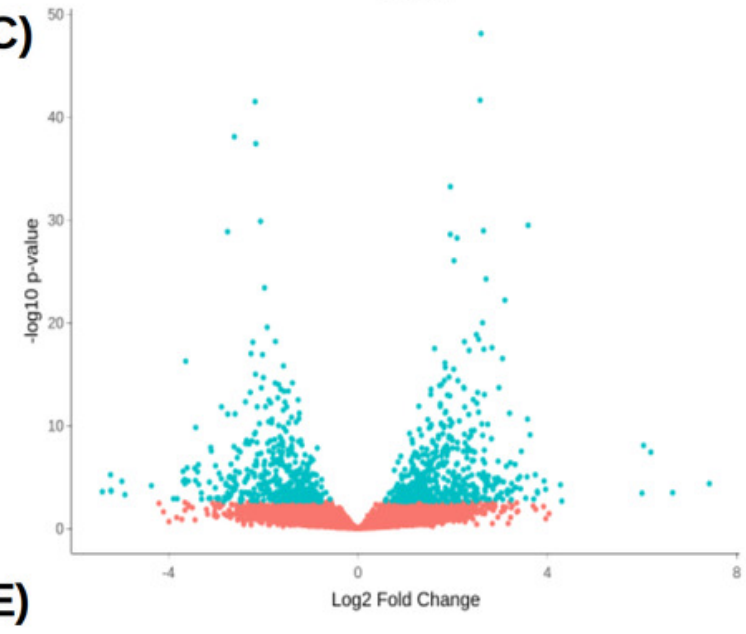

E)

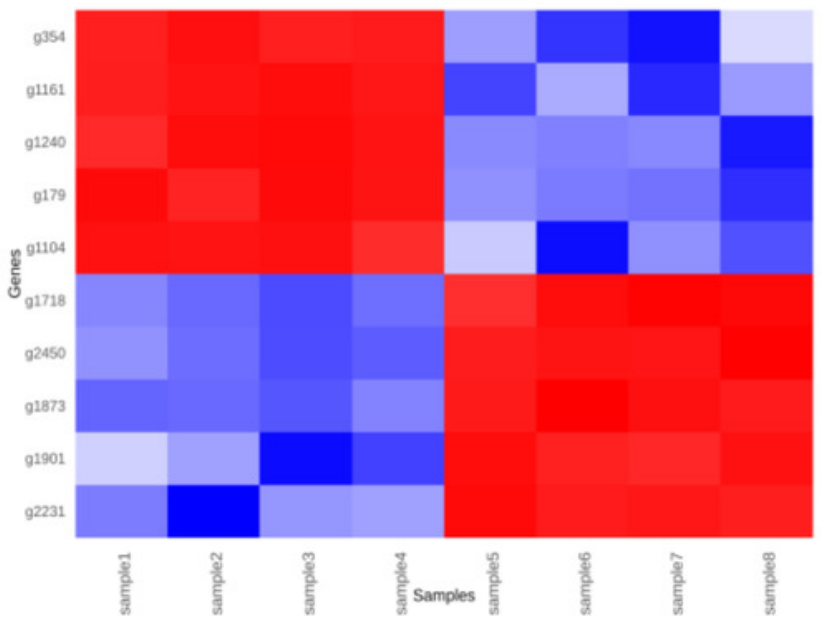

B)

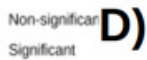

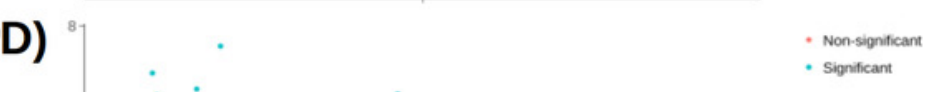

a Domregulated

- Upregulated
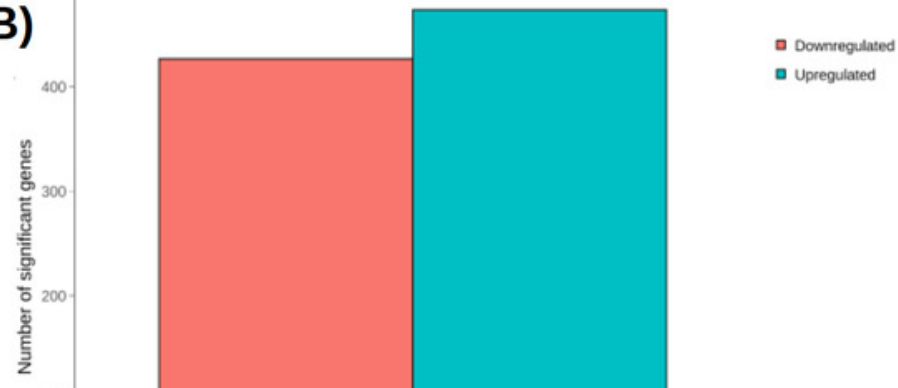
Figure 3

An overview of BingleSeq's 'Clustering' tab with A) clustering customization options, B) general tips and advice for the selected clustering package (in this case SC3), and clustering-related visualization options: C) A PC elbow plot, D) a PC heatmap show 
A)

BingleSeq Load Data Quality Control Normalization Clustering Diflerential Expression Compare DE Methods Functional Annotation

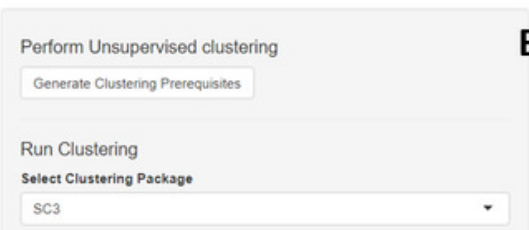

B) Unsuperised Clustering with SCO3:

SC3's inbuin fittering options enable further reducion of noise by filtering out

instart parameter enables control over the number of random initas centroids used in clustering and hence computational time. By default, this parameter is set to 1000 when working with less than 2000 cells and to 50 when working with more than 2000 cells SC 3 is magnitudes slower than the other approaches and appropriately setting instart is essental.

$\mathrm{SC} 3$

Filter genes below minimum percent of dropouts
$\begin{array}{ll}0 & \text { C) }\end{array}$

Filter genes above maximum percent of dropouts

100

Random sets used in clustering (nStart)

50

- Estimate Cluster Number

Cluster Data

Visualize Results

Choose Plot Type

- Ebow plot
Dimensions Heatmap

Oimensions

ISNE Plot

Generate Plot

Note that SC3 enables the number of clusters to be spectied or estimated.

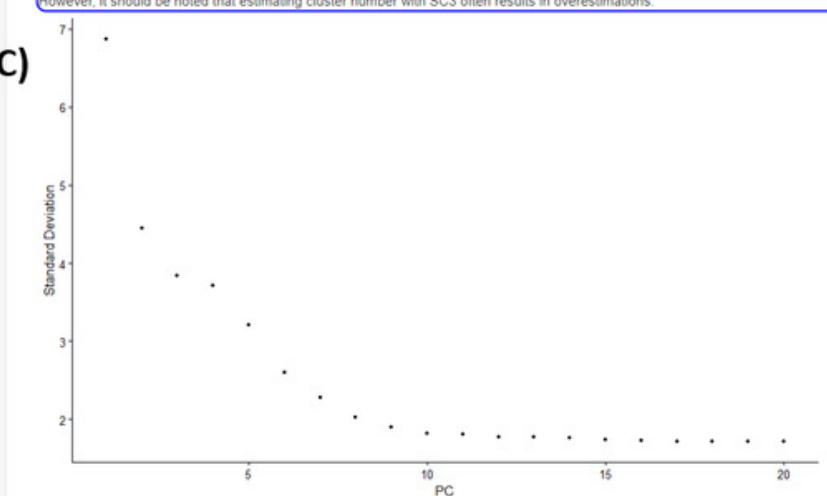

Save Object

Number of estimated clusters 1s: ?

\$D Download Plot

D)
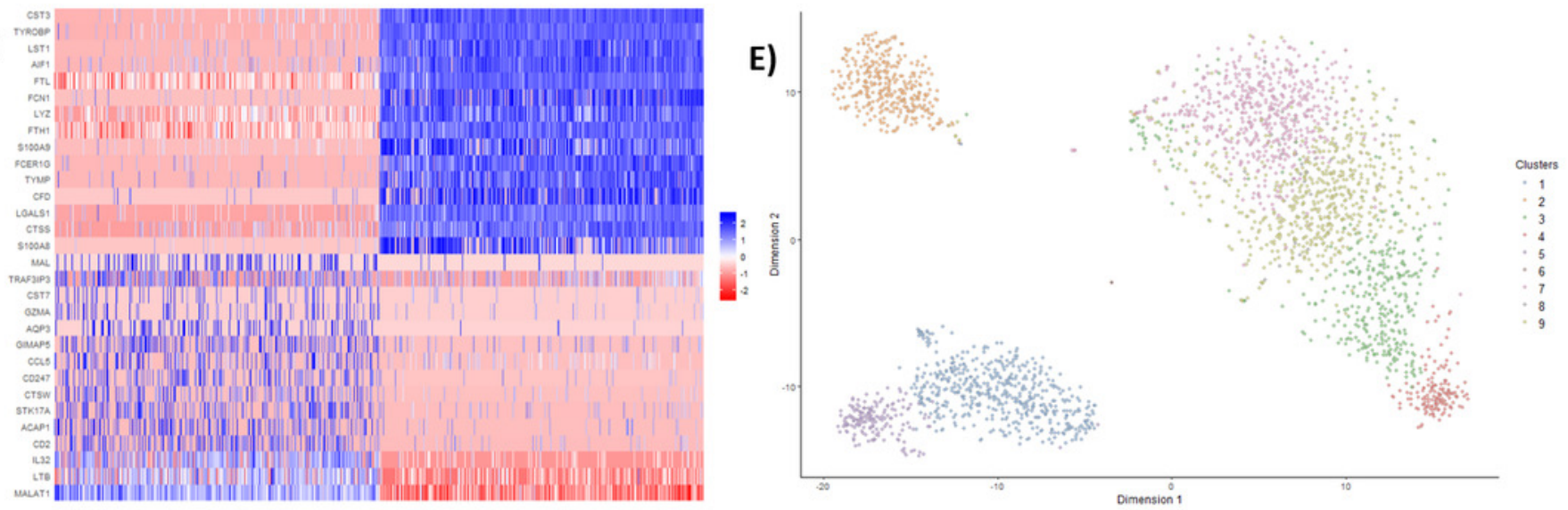

F)

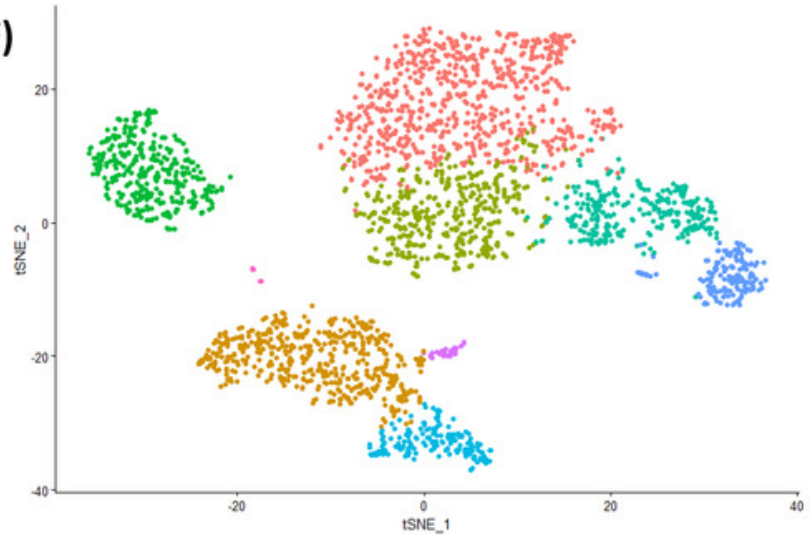

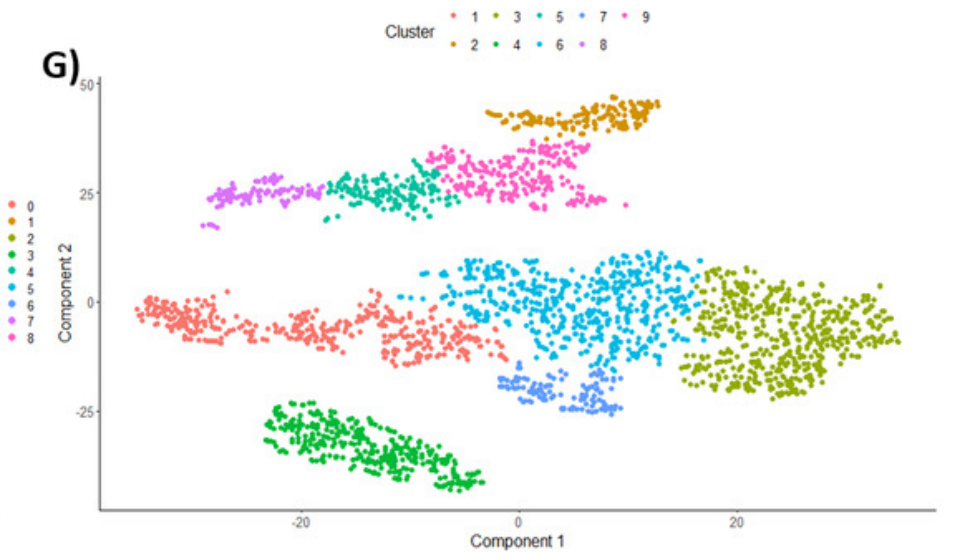


Figure 4

A) Heatmap showing the top 10 genes for each cluster in the 2700 PBMCs dataset, while Violin $B$ ), Feature $C$ ), and Ridge $D$ ) plots are shown for MS4A1 gene - a biomarker of $B$ lymphocytes. Note that these DE visualization options are available in BingleSeq an

A)

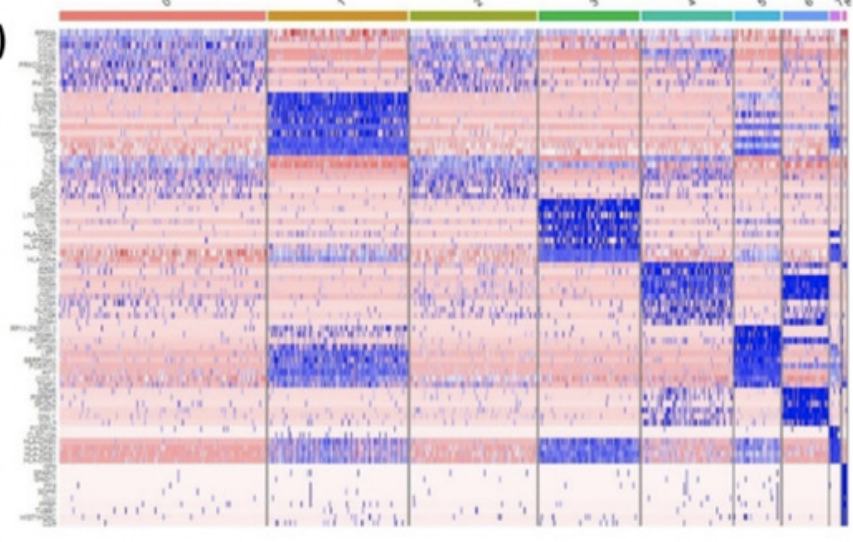

C)

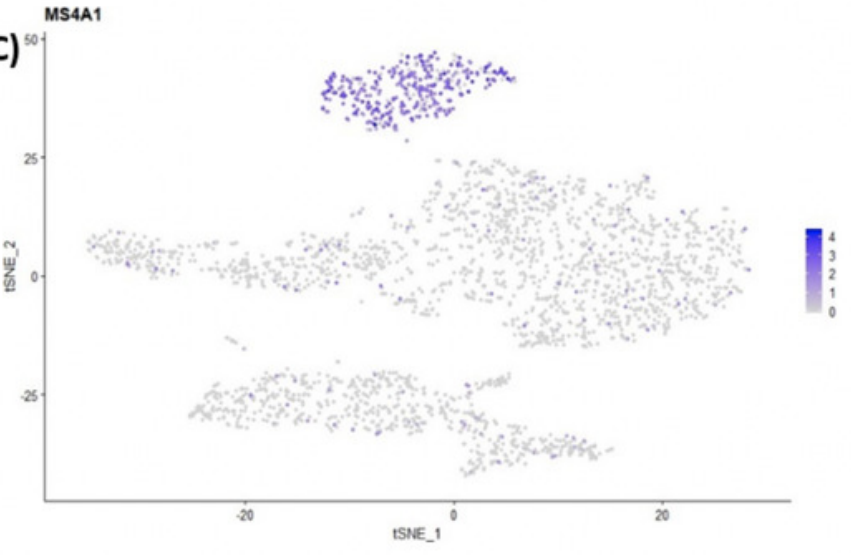

B)

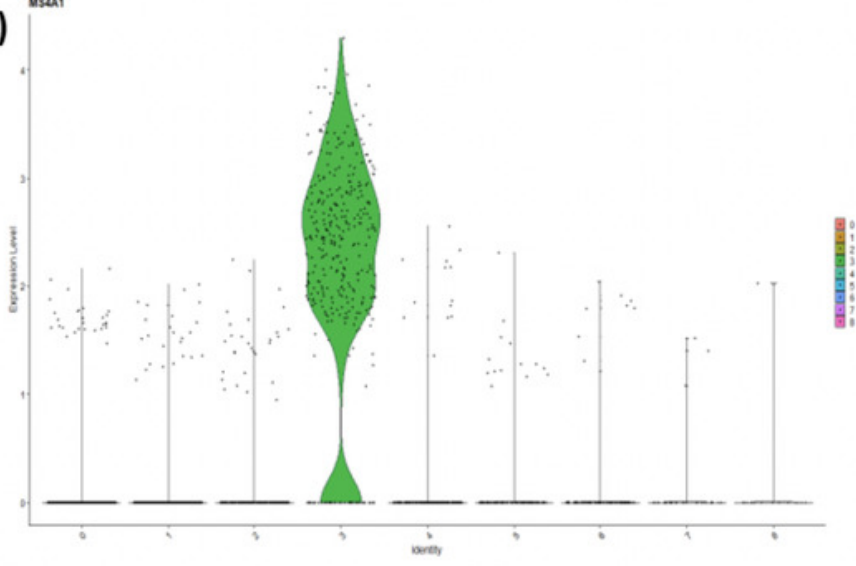

D)

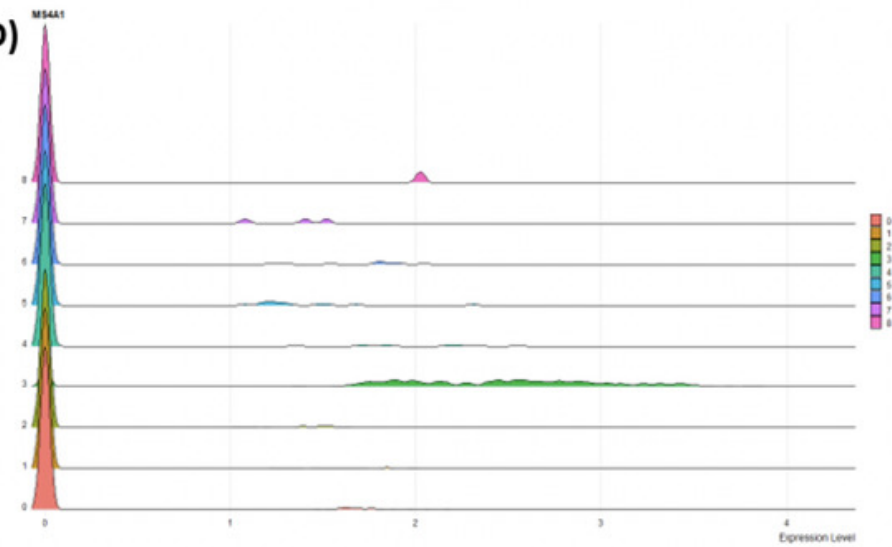

